\title{
Conservation Laws and Lumped System Dynamics
}

\author{
A.J. van der Schaft, B.M. Maschke
}

\section{Introduction}

Physical systems modeling, aimed at network modeling of complex multi-physics systems, has especially flourished in the fifties and sixties of the 20-th century, see e.g. $[11,4]$ and references provided therein. With the reinforcement of the 'systems' legacy in Systems \& Control, the growing recognition that 'control' is not confined to developing algorithms for processing the measurements of the system into control signals (but instead is concerned with the design of the total controlled system), and facing the complexity of modern technological and natural systems, systematic methods for physical systems modeling of large-scale lumped- and distributedparameter systems capturing their basic physical characteristics are needed more than ever.

In this paper we are concerned with the development of a systematic framework for modeling multi-physics systems which is directly based on conservation laws. Modeling based on conservation laws is prevalent in a distributed-parameter context in areas such as fluid dynamics and hydraulic systems, chemical and thermodynamical systems [2], as well as electromagnetism, but is also underlying the basic structure of lumped-parameter systems such as electrical circuits. While the natural framework for formulating Kirchhoff's laws for electrical circuits is the circuit graph we will show in this paper how distributed-parameter conservation laws can be discretized by using the proper generalization of the notion of graph to 'higherdimensional networks', called $k$-complexes in algebraic topology. Furthermore, we show how these discretized conservation laws define a power-conserving intercon-

A.J. van der Schaft

Institute of Mathematics and Computing Science, University of Groningen, PO Box 407, 9700 AK, the Netherlands, e-mail: A.J.van.der.Schaft@rug.nl

B.M. Maschke

Lab. d'Automatique et de Genie des Procédés, Université Claude Bernard Lyon-1, F-69622 Villeurbanne, Cedex, France, e-mail: maschke@lagep.univ-lyon1.fr 
nection structure, called a Dirac structure, which, when combined with the (discretized) constitutive relations, defines a finite-dimensional port-Hamiltonian system $[14,13,5]$.

In previous work [15] we have laid down a framework for formulating conservation laws described by partial differential equations as infinite-dimensional port-Hamiltonian systems. Furthermore, in [8] we have shown how such infinitedimensional port-Hamiltonian systems can be spatially discretized to finite-dimensional port-Hamiltonian systems by making use of mixed finite-element methods. In this paper we show how alternatively we can directly spatially 'lump' the dynamics described by conservation laws in a structure-preserving manner, again obtaining a finite-dimensional port-Hamiltonian system description. This approach also elucidates the concept of the spatial system boundary, and leads to the notion of distributed terminals.

This paper is a follow-up of our previous paper [16]. Older references in this spirit include [10, 12].

\section{Kirchhoff's laws on graphs and circuit dynamics}

In this section we recall the abstract formulation of Kirchhoff's laws on graphs, dating back to the historical work of Kirchhoff [9], as can be found e.g. in [1, 3]. In order to deal with open electrical circuits we define open graphs, and we show how Kirchhoff's laws on open graphs define a power-conserving interconnection structure, called a Dirac structure, between the currents through and the voltages over the edges of the graph, and the boundary currents and potentials. This enables us to describe the circuit dynamics as a port-Hamiltonian system.

\subsection{Graphs}

An oriented graph $^{1} \mathscr{G}$, see e.g. [3], consists of a finite set $\mathscr{V}$ of vertices and a finite set $\mathscr{E}$ of directed edges, together with a mapping from $\mathscr{E}$ to the set of ordered pairs of $\mathscr{V}$. Thus to any branch $e \in \mathscr{E}$ there corresponds an ordered pair $(v, w) \in \mathscr{V}^{2}$ representing the initial vertex $v$ and the final vertex $w$ of this edge. An oriented graph is completely specified by its incidence matrix $B$, which is an $\bar{v} \times \bar{e}$ matrix, $\bar{v}$ being the number of vertices and $\bar{e}$ being the number of edges, with $(i, j)$-th element $b_{i j}$ equal to 1 if the $j$-th edge is an edge towards vertex $i$, equal to -1 if the $j$-th edge is an edge originating from vertex $i$, and 0 otherwise.

Given an oriented graph we define its vertex space $\Lambda_{0}$ as the real vector space of all functions from $\mathscr{V}$ to $\mathbb{R}$. Clearly $\Lambda_{0}$ can be identified with $\mathbb{R}^{\bar{v}}$. Furthermore, we

${ }^{1}$ In fact, we will be considering multi-graphs since we allow for the existence of multiple branches between the same pair of vertices. 
define its edge space $\Lambda_{1}$ as the vector space of all functions from $\mathscr{E}$ to $\mathbb{R}$. Again, $\Lambda_{1}$ can be identified with $\mathbb{R}^{\bar{e}}$.

In the context of an electrical circuit $\Lambda_{1}$ will be the vector space of currents through the edges in the circuit. The dual space of $\Lambda_{1}$ will be denoted by $\Lambda^{1}$, and defines the vector space of voltages across the edges. (We have highlighted the words 'through' and 'across' to refer to the classical use of 'through' and 'across' variables, see e.g. [11].) Furthermore, the duality product $\langle V \mid I\rangle=V^{T} I$ of a vector of currents $I \in \Lambda_{1}$ with a vector of voltages $V \in \Lambda^{1}$ is the total power over the circuit. Similarly, the dual space of $\Lambda_{0}$ is denoted by $\Lambda^{0}$ and defines the vector space of potentials at the vertices.

Remark 1. Since $\Lambda_{0}$ and $\Lambda_{1}$ have a canonical basis corresponding to the individual vertices, respectively edges, there is a standard Euclidean inner product on both spaces, and thus both $\Lambda^{0}$ and $\Lambda^{1}$ can be identified with $\Lambda_{0}$, respectively $\Lambda_{1}$, such that the duality product becomes this standard inner product. In situations to be treated later on this will not necessarily be the case.

The incidence matrix $B$ can be also regarded as the matrix representation of a linear map (denoted by the same symbol)

$$
B: \Lambda_{1} \rightarrow \Lambda_{0}
$$

called the incidence operator or (boundary operator). Its adjoint map is denoted in matrix representation as

$$
B^{T}: \Lambda^{0} \rightarrow \Lambda^{1},
$$

and is called the co-incidence (or co-boundary) operator.

\subsection{Kirchhoff's laws for graphs}

Consider an oriented graph $\mathscr{G}$ specified by its incidence operator $B$. Kirchhoff's laws associated with the graph are expressed as follows. Kirchhoff's current laws (KCL) are given as

$$
I \in \operatorname{ker} B,
$$

while Kirchhoff's voltage laws (KVL) take the form

$$
V \in \operatorname{im} B^{T} .
$$

A graph theoretic interpretation of Kirchhoff's current and voltage laws can be given as follows [3]. The kernel of the incidence operator $B$ is the cycle space $Z \subset \Lambda_{1}$ of the graph, while the image $U \subset \Lambda^{1}$ of the co-incidence operator $B^{T}$ is its cut space (or, co-cycle space). Since $\operatorname{ker} B=\left(\operatorname{im} B^{T}\right)^{\perp}$ (with ${ }^{\perp}$ denoting the orthogonal complement with respect to the duality product between the dual spaces $\Lambda_{1}$ and $\Lambda^{1}$ ) the cycle space is the orthogonal complement of the cut space. 
This leads to the equivalent way of formulating Kirchhoff's current laws as the fact that the total current $I$ along any cut is equal to zero, since $I \in \operatorname{ker} B$ is equivalent to $I$ being orthogonal to the cut space $U$. The simplest elements of the cut space $U$ (which in fact are spanning the linear space $U$ ) are the cuts given by all edges starting from or terminating on a single vertex $v$. Kirchhoff's current laws for these cut sets mean nothing else than the expression that the currents entering or leaving any vertex $v$ sum up to zero. Indeed, if $v$ is numbered as the $i$-th vertex then the $i$-the equation in the linear set of equations $B I=0$ is precisely this.

On the other hand, since $V \in \operatorname{im} B^{T}$ is equivalent to $V$ being orthogonal to the cycle space $Z$, Kirchhoff's voltage laws can be equivalently described as the fact that the total voltage over every cycle is zero.

The difference between Kirchhoff's current and voltage laws is also reflected by writing Kirchhoff's voltage laws as

$$
V=B^{T} \psi
$$

for some vector $\psi \in \Lambda^{0}$, which has the physical interpretation of being the vector of potentials at every vertex. Hence Kirchhoff's voltage laws express that the voltage distribution $V$ over the edges of the graph corresponds to a potential distribution over the vertices.

Of course, Tellegen's theorem automatically follows from Kirchhoff's laws. Indeed, take any current distribution $I$ satisfying Kirchhoff's current laws $B I=0$, and any voltage distribution $V$ satisfying Kirchhoff's voltage laws $V=B^{T} \psi$. Then,

$$
V^{T} I=\psi^{T} B I=0
$$

In particular, Tellegen's theorem implies that for any actual current and voltage distribution over the circuit the total power $V^{T} I$ is equal to zero.

We summarize the Kirchhoff behavior $\mathscr{B}_{K}(\mathscr{G})$ of a graph $\mathscr{G}$ with incidence ma$\operatorname{trix} B$ as

$$
\mathscr{B}_{K}(\mathscr{G}):=\left\{(I, V) \in \Lambda_{1} \times \Lambda^{1} \mid I \in \operatorname{ker} B, V \in \operatorname{im} B^{T}\right\}
$$

It immediately follows that the Kirchhoff behavior defines a Dirac structure. Recall $[6,14,13]$ that a subspace $D \subset V \times V^{*}$ for some vector space $V$ defines a Dirac structure if $D=D^{\text {orth }}$ where ${ }^{\text {orth }}$ denotes the orthogonal complement with respect to the indefinite inner product $<<,>>$ on $V \times V^{*}$ defined as

$$
<<\left(v_{1}, v_{1}^{*}\right),\left(v_{2}, v_{2}^{*}\right)>>:=<v_{1}^{*}\left|v_{2}>+<v_{2}^{*}\right| v_{1}>,
$$

with $v_{1}, v_{2} \in V, v_{1}^{*}, v_{2}^{*} \in V^{*}$, where $<\mid>$ denotes the duality product between $V$ and $V^{*}$. 


\subsection{Kirchhoff's laws for open graphs}

Although in Kirchhoff's original treatment of circuits and graphs external currents entering the vertices of the graph were an indispensable notion, this has not been articulated very well in the subsequent formalization of circuits and graphs ${ }^{2}$. Hence a reinforcement of this systems point of view is definitely in order.

We will do so by extending the notion of graph to open graph. An open graph $\mathscr{G}$ is obtained from an ordinary graph with set of vertices $\mathscr{V}$ by identifying a subset $\mathscr{V}_{b} \subset \mathscr{V}$ of boundary vertices. The interpretation of $\mathscr{V}_{b}$ is that these are the vertices that are open to interconnection (e.g., with other graphs). The remaining subset $\mathscr{V}_{i}:=$ $\mathscr{V}-\mathscr{V}_{b}$ are the internal vertices of the open graph.

Remark 2. Another way of defining open graphs is by identifying some of the edges to be the boundary edges (open to interconnection). Such a definition is straightforward, and we will not elaborate on this. The distinction between the definitions of an open graph using boundary vertices or boundary edges is analogous to the difference between boundary control of distributed-parameter systems and distributed control; see also Section 3.

Kirchhoff's current laws apply to an open graph $\mathscr{G}$ in a different manner than to an ordinary graph, since the ordinary Kirchhoff's current laws would imply that the sum of the currents over all edges incident on a boundary vertex is zero, which is not what we want for interconnection. Furthermore, by Tellegen's theorem, the ordinary KCL would imply that the total power in the circuit is equal to zero, thus implying that there cannot be any ingoing or outgoing power flow. Hence we have to modify Kirchhoff's current laws by requiring that the incidence operator $B$ maps the vector of currents $I$ to a vector that has zero components corresponding to the internal vertices, while for the boundary vertices the image is equal to (minus) the boundary current $I_{b}$. Decomposing the incidence operator $B$ as $\left[\begin{array}{l}B_{i} \\ B_{b}\end{array}\right]$ with $B_{i}$ the part of the incidence operator corresponding to the internal vertices, and $B_{b}$ the part corresponding to the boundary vertices, we thus arrive at

$$
B_{i} I=0, \quad B_{b} I=-I_{b}, \quad \mathrm{KCL}
$$

Here the vector $I_{b}$ is belonging to the vector space $\Lambda_{b}$ of functions from the boundary vertices $\mathscr{V}_{b}$ to $\mathbb{R}$ (which is identified with $\mathbb{R}^{\bar{v}_{b}}$, with $\bar{v}_{b}$ the number of boundary vertices $)^{3}$.

Kirchhoff's voltage laws (KVL) remain unchanged, and will be written as

\footnotetext{
${ }^{2}$ Unfortunately, this holds for many formalizations of physical theories over the last century. A proper theory of mechanics should include external forces from the very start, instead of restricting itself to closed mechanical systems. Thermodynamics cannot be properly formalized without taking interaction with other systems into account.

${ }^{3}$ Alternatively, open graphs can be defined by attaching 'one-sided open edges' (properly called leaves) to every boundary vertex in $\mathscr{V}_{b}$. Then the elements of the vector $I_{b}$ are the currents through these leaves, see also [17].
} 


$$
V=B^{T} \psi=B_{i}^{T} \psi_{i}+B_{b}^{T} \psi_{b}, \quad \mathrm{KVL}
$$

where $\psi_{i}$ denotes the vector of the potentials at the internal vertices and $\psi_{b}$ the vector of potentials at the boundary vertices. Note that $\psi_{b} \in \Lambda^{b}$ (where we define $\Lambda^{b}$ to be the dual of the space of boundary currents $\Lambda_{b}$ ). This results in the following Kirchhoff behavior for an open graph $\mathscr{G}$ :

$$
\begin{aligned}
B_{K}(\mathscr{G}):= & \left\{\left(I, V, I_{b}, \psi_{b}\right) \in \Lambda_{1} \times \Lambda^{1} \times \Lambda_{b} \times \Lambda^{b} \mid\right. \\
& \left.B_{i} I=0, B_{b} I=-I_{b}, \exists \psi_{i} \text { s.t. } V=B_{i}^{T} \psi_{i}+B_{b}^{T} \psi_{b}\right\}
\end{aligned}
$$

By computing as before, cf. (4), the total power over the graph we now obtain

$$
V^{T} I=\psi_{i}^{T} B_{i} I+\psi_{b}^{T} B_{b} I=-\psi_{b}^{T} I_{b}
$$

Thus, for open graphs the total power $V^{T} I$ is equal to the outgoing power $-\psi_{b}^{T} I_{b}$. This will lead to the following characterization of the Kirchhoff behavior of open graphs as Dirac structures.

Proposition 1. Let $\mathscr{G}$ be an open graph with incidence matrix $B=\left[\begin{array}{l}B_{i} \\ B_{b}\end{array}\right]$. Then its Kirchhoff behavior $\mathscr{B}_{K}(\mathscr{G})$ is a Dirac structure.

Proof. As shown in (9), $V^{T} I+\psi_{b}^{T} I_{b}=0$. By considering $I=I_{1}+I_{2}, V=V_{1}+$ $V_{2}, I_{b}=I_{b 1}+I_{b 2}, \psi=\psi_{1}+\psi_{2}$, with $\left(I_{j}, V_{j}, I_{b j}, \psi_{j}\right) \in \mathscr{B}_{K}(\mathscr{G}), j=1,2$, it follows that

$$
\ll\left(I_{1}, V_{1}, I_{b 1}, \psi_{1}\right),\left(I_{2}, V_{2}, I_{b 2}, \psi_{2}\right) \gg=0,
$$

which implies that $\mathscr{B}_{K}(\mathscr{G}) \subset\left(\mathscr{B}_{K}(\mathscr{G})\right)^{\text {orth }}$.

For showing the reverse inclusion, consider a quadruple $\left(I, V, I_{b}, \psi_{b}\right) \in\left(\mathscr{B}_{K}(\mathscr{G})\right)^{\text {orth }}$, that is,

$$
V^{T} \bar{I}+\bar{V}^{T} I+\psi_{i}^{T} \bar{I}_{b}+\bar{\psi}_{b}^{T} I_{b}=0
$$

for all $\left(\bar{I}, \bar{V}, \bar{I}_{b}, \bar{\psi}_{b}\right)$ satisfying

$$
B_{i} \bar{I}=0, B_{b} \bar{I}=-\bar{I}_{b}, \bar{V}=B_{i}^{T} \bar{\psi}_{i}+B_{b}^{T} \bar{\psi}_{b}, \quad \text { for some } \bar{\psi}_{i} .
$$

Writing out (10) we obtain

$$
\begin{aligned}
0 & =V^{T} \bar{I}+\bar{\psi}_{i}^{T} B_{i} I+\bar{\psi}_{b}^{T} B_{b} I-\bar{\psi}_{b}^{T} B_{i} I+\bar{\psi}_{b}^{T} I_{b} \\
& =\left(V-B_{b}^{T} \psi_{b}\right)^{T} \bar{I}+\bar{\psi}_{i}^{T} B_{i} I+\bar{\psi}_{b}^{T}\left(B_{b} I+I_{b}\right)
\end{aligned}
$$

for all $\bar{\psi}_{i}, \bar{\psi}_{b}$ and all $\bar{I}$ satisfying $B_{i} \bar{I}=0$. It follows that $B_{i} I=0, B_{b} I+I_{b}=0$, and $V-B_{b}^{T} \psi_{b} \in \operatorname{im} B_{i}^{T}$, or equivalently $V-B_{b}^{T} \psi_{b}=B_{i}^{T} \psi_{i}$ for some $\psi_{i}$. 


\subsection{Constraints on boundary currents and invariance of boundary potentials}

It is a well-known property [3] of any incidence matrix $B$ that

$$
\mathbb{1}^{T} B=0
$$

where $\mathbb{1}$ denotes the vector with all components equal to 1 . From this property it follows that the rank of the incidence matrix $B$ is at most $\bar{v}-1$. In fact, the rank is given as [3] $\operatorname{rank} B=\bar{v}-k_{\mathscr{G}}$, where $k_{\mathscr{G}}$ is the number of components ${ }^{4}$ of the graph $\mathscr{G}$. (Thus $\operatorname{rank} B=\bar{v}-1$ for a connected graph.) By (6) it follows that

$$
0=\mathbb{1}^{T} B I=\mathbb{1}_{b}^{T} B_{b} I=-\mathbb{1}_{b}^{T} I_{b}=-\sum_{v_{b}} I_{v_{b}}
$$

with $\mathbb{1}_{b}$ denoting the vector with all ones of dimension equal to the number of boundary vertices, and where the summation is over all boundary vertices $v_{b} \in \mathscr{V}_{b}$. Hence the boundary part of the Kirchhoff behavior of an open graph is constrained by the obvious fact that all boundary currents sum up to zero. Dually, we may always add to the vector of potentials $\psi$ the vector $\mathbb{1}$ leaving invariant the vector of voltages $V=B^{T} \psi$. Hence, to the vector of boundary potentials $\psi_{b}$ we may always add the vector $\mathbb{1}_{b}$. Summarizing we arrive at a similar statement as in [17]):

Proposition 2. Consider an open graph $\mathscr{G}$ with Kirchhoff behavior $\mathscr{B}_{K}(\mathscr{G})$. Then for each $\left(I, V, I_{b}, \psi_{b}\right) \in \mathscr{B}_{K}(\mathscr{G})$ it holds that

$$
\mathbb{1}_{b}^{T} I_{b}=0
$$

while for any constant $c \in \mathbb{R}$

$$
\left(I, V, I_{b}, \psi_{b}+c \mathbb{1}_{b}\right) \in \mathscr{B}_{K}(\mathscr{G})
$$

This implies that we may restrict the dimension of the space of external variables $\Lambda_{b} \times \Lambda^{b}$ by two. Indeed, we may define

$$
\Lambda_{b \mathrm{red}}:=\left\{I_{b} \in \Lambda_{b} \mid I_{b} \in \operatorname{ker} \mathbb{1}_{b}^{T}\right\}
$$

and its dual space

$$
\Lambda_{\mathrm{red}}^{b}:=\left(\Lambda_{b \mathrm{red}}\right)^{*}=\Lambda^{b} / \operatorname{im} \mathbb{1}_{b}
$$

It is rather straightforward to show that the Kirchhoff behavior $\mathscr{B}_{K}(\mathscr{G})$ reduces to a linear subspace of the reduced space $\Lambda_{1} \times \Lambda^{1} \times \Lambda_{\text {bred }} \times \Lambda_{\text {red }}^{b}$, which is also a Dirac structure. A circuit interpretation of this reduction is that we may consider one of the boundary vertices, say the first one, to be the reference ground vertex, and that we may reduce the vector of boundary potentials $\psi_{b}=\left(\psi_{b 1}, \cdots, \psi_{b \bar{v}_{b}}\right)$ to a vector

${ }^{4} \mathrm{~A}$ component is a maximal subgraph which is connected, that is, every two vertices are linked by a path of, -non-oriented-, edges. 
of voltages $\left(\psi_{b 2}-\psi_{b 1}, \cdots, \psi_{b \bar{v}_{b}}-\psi_{b 1}\right)$. A graph-theoretical interpretation is that instead of the incidence operator $B$ we consider the restricted incidence operator [1].

For a graph $\mathscr{G}$ with more than one connected component the above holds for each connected component. It follows that there are as many independent constraints on the boundary currents $I_{b}$ as the number of the connected components of the open graph $\mathscr{G}$. Dually, the space of allowed boundary potentials $\psi_{b}$ is invariant under translation by as many independent vectors $\mathbb{1}_{b}$ as the number of connnected components.

A complementary view on the fact that the sum of the boundary currents is equal to zero and the boundary potentials are invariant under translation along $\mathbb{1}_{b}$ is the fact that we may close an open graph $\mathscr{G}$ to an ordinary graph $\overline{\mathscr{G}}$. Consider first the case that $\mathscr{G}$ is connected. Then we may add one virtual ('ground') vertex $v_{0}$, and virtual edges from this virtual vertex to every boundary vertex $v_{b} \in \mathscr{V}_{e}$, in such a manner that the Kirchhoff behavior of this graph $\overline{\mathscr{G}}$ extends the Kirchhoff behavior of the open graph $\mathscr{G}$. In fact, to the virtual vertex $v_{0}$ we may associate an arbitrary potential $\psi_{v_{0}}$ (a ground-potential), and we may rewrite the righthand-side of (9) as (since $\sum_{v_{b}} I_{v_{b}}=0$ )

$$
-\sum_{v_{b}}\left(\psi_{v_{b}}-\psi_{v_{0}}\right) I_{v_{b}}=-\sum_{v_{b}} V_{v_{b}} I_{v_{b}}
$$

where $V_{v_{b}}:=\psi_{v_{b}}-\psi_{v_{0}}$ and $I_{v_{b}}$ denotes the voltage across and the current through the virtual edge towards the boundary vertex $v_{b}$.

If the open graph $\mathscr{G}$ consists of more than one component, then one extends the graph by adding a virtual vertex to every component containing boundary vertices.

\subsection{Interconnection of open graphs}

Consider two open graphs $\mathscr{G}^{j}$ with boundary operators $B^{j}=\left[\begin{array}{l}B_{i}^{j} \\ B_{b}^{j}\end{array}\right], j=1,2$. Interconnection is done by identifying some of their boundary vertices, and equating (up to a minus sign) the boundary potentials and currents corresponding to these boundary vertices.

For simplicity we consider the case that we can equate all their boundary vertices with each other, resulting in an ordinary (closed) graph with set of vertices $\mathscr{V}_{i}^{1} \cup$ $\mathscr{V}_{i}^{2} \cup \mathscr{V}$, where $\mathscr{V}_{i}:=\mathscr{V}_{b}^{1}=\mathscr{V}_{b}^{2}$ denotes the set of shared boundary vertices. The incidence operator $B$ of this interconnected graph is given as

$$
B=\left[\begin{array}{cc}
B_{i}^{1} & 0 \\
0 & B_{i}^{2} \\
B_{b}^{1} & B_{b}^{2}
\end{array}\right]
$$

corresponding to the following interconnection constraints on the boundary potentials and currents 


$$
\psi_{b}^{1}=\psi_{b}^{2}, \quad I_{b}^{1}+I_{b}^{2}=0
$$

Of course, several extensions are possible. For example, one may still retain the shared vertices $\mathscr{V}_{b}:=\mathscr{V}_{b}^{1}=\mathscr{V}_{b}^{2}$ as being boundary vertices (instead of internal vertices as above) by extending (15) to

$$
\psi_{b}^{1}=\psi_{b}^{2}=\psi_{b}, \quad I_{b}^{1}+I_{b}^{2}+I_{b}=0
$$

with $I_{b}, \psi_{b}$ the boundary currents and potentials of the interconnected graph.

\subsection{Constitutive relations and port-Hamiltonian circuit dynamics}

The dynamics of an RLC-circuit is defined, on top of Kirchhoff's laws for its circuit graph, by the constitutive relations of its elements (in this case, capacitors, inductors and resistors). They specify for each edge $e$ a relation between the current $I_{e}$ through and the voltage $V_{e}$ across the edge. The simplest case is a resistive relation between $I_{e}$ and $V_{e}$ such that $V_{e} I_{e} \leq 0$. In particular, a linear resistor at edge $e$ is specified by a relation $V_{e}=-R_{e} I_{e}$ with $R_{e} \geq 0$. In the case of a capacitive relation one defines an additional energy variable $Q_{e}$ (denoting the charge) together with a real function $H_{C e}\left(Q_{e}\right)$ denoting the electric energy stored in the capacitor. The constitutive relations for a capacitor at edge $e$ are given by

$$
\dot{Q}_{e}=-I_{e}, \quad V_{e}=\frac{d H_{C e}}{d Q_{e}}\left(Q_{e}\right)
$$

Alternatively, in the case of an inductor one specifies the magnetic energy $H_{L e}\left(\Phi_{e}\right)$, where $\Phi_{e}$ denotes the magnetic flux linkage, together with the dynamic relations

$$
\dot{\Phi}_{e}=-V_{e}, \quad I_{e}=\frac{d H_{L e}}{d \Phi_{e}}\left(\Phi_{e}\right)
$$

Substituting these constitutive relations into the Kirchhoff behavior $\mathscr{B}_{K}(\mathscr{G})$ (which is a Dirac structure) results in a port-Hamiltonian ${ }^{5}$ system, see e.g. [14, 13], given by

$$
\left(\left(I_{C}, I_{L}, I_{R}\right),\left(V_{C}, V_{L}, V_{R}\right), I_{b}, \psi_{b}\right) \in \mathscr{B}_{K}(\mathscr{G})
$$

where the vectors $I_{C}, I_{L}, I_{R}, V_{C}, V_{L}, V_{R}$ denote the currents, respectively voltages, corresponding to the capacitors, inductors, and resistors, related as

\footnotetext{
${ }^{5}$ Strictly speaking, the terminology 'port-Hamiltonian' is not completely appropriate since this assumes that the system boundary consists of ports, that is pairs of vertices with boundary variables being the currents through and the voltages across the edge corresponding to each port. Nevertheless, the mathematical structure and system description remains the same. Furthermore, by reducing the Kirchhoff behavior as above, or alternatively by extending it through the addition of a ground vertex, the boundary variables become true port variables.
} 


$$
I_{C}=-\dot{Q}, V_{C}=\frac{\partial H_{C}}{\partial Q}(Q), \quad V_{L}=-\dot{\Phi}, I_{L}=\frac{\partial H_{L}}{\partial \Phi}(\Phi),
$$

where $Q$ denotes the vector of charges at the capacitors, a $\Phi$ denotes the vector of fluxes at the inductors, $H_{C}$ and $H_{L}$ denote the total electric and magnetic energies, and where moreover the vectors $I_{R}, V_{R}$ satisfy a resistive relation.

Example 1. Let us consider an LC-circuit (for simplicity without boundary vertices). We will start by decomposing the circuit graph $\mathscr{G}$ as the interconnection of a graph corresponding to the capacitors and a graph corresponding to the inductors. Define $\hat{V}$ as the set of all vertices that are adjacent to at least one capacitor as well as to at least one inductor. Then split the circuit graph into an open circuit graph $\mathscr{G}^{C}$ corresponding to the capacitors and an open circuit graph $\mathscr{G}^{L}$ corresponding to the inductors, both with set of boundary vertices $\hat{V}$. Denote the incidence matrices of these two circuit graphs by

$$
B^{C}:=\left[\begin{array}{l}
B_{i}^{C} \\
B_{b}^{C}
\end{array}\right], B^{L}:=\left[\begin{array}{l}
B_{i}^{L} \\
B_{b}^{L}
\end{array}\right]
$$

Assuming for simplicity that all capacitors and inductors are linear we arrive at the following equations for the $C$-circuit

$$
\begin{aligned}
B_{b}^{C} \dot{Q} & =I_{b}^{C}, B_{i}^{C} \dot{Q}=0 \\
B_{b}^{C T} \psi_{b}^{C} & =C^{-1} Q-B_{i}^{C T} \psi_{i}^{C}
\end{aligned}
$$

with $C$ the diagonal matrix with diagonal elements corresponding to the capacitances of the capacitors, and for the $L$-circuit

$$
\begin{aligned}
\dot{\Phi} & =B_{b}^{L T} \psi_{b}^{L}+B_{i}^{L T} \psi_{i}^{L} \\
0 & =B_{i}^{L} L^{-1} \Phi \\
I_{b}^{L} & =-B_{i}^{L} \Phi
\end{aligned}
$$

with $L$ the diagonal matrix of inductances.

The equations of the $L C$-circuit are obtained by imposing the interconnection constraints $\psi_{b}^{C}=\psi_{b}^{L}=: \psi_{i}$ and $I_{b}^{C}+I_{b}^{L}=0$. By eliminating the boundary currents $I_{b}^{C}, I_{b}^{L}$ one arrives at the equations

$$
\begin{aligned}
& {\left[\begin{array}{cc}
B_{i}^{C} & 0 \\
0 & B_{i}^{L} \\
B_{b}^{C} & B_{b}^{L}
\end{array}\right]\left[\begin{array}{c}
-\dot{Q} \\
L^{-1} \Phi
\end{array}\right]=0} \\
& {\left[\begin{array}{c}
C^{-1} Q \\
-\dot{\Phi}
\end{array}\right]=\left[\begin{array}{ccc}
B_{i}^{C T} & 0 & B_{b}^{C T} \\
0 & B_{i}^{L T} & B_{b}^{L T}
\end{array}\right]\left[\begin{array}{l}
\psi_{i}^{C} \\
\psi_{i}^{L} \\
\psi_{i}
\end{array}\right]}
\end{aligned}
$$




\section{Conservation laws on higher-dimensional complexes}

In this section we will extend the formalization of conservation laws on graphs as expressed by Kirchhoff's laws to higher-dimensional networks. In particular, this will allow us to systematically spatially discretize distributed-parameter physical systems to finite-dimensional lumped-parameter systems, represented in portHamiltonian form.

\subsection{Kirchhoff behavior on k-complexes}

An oriented graph with incidence matrix $B$ is a typical example of what is called in algebraic topology a 1-complex. Indeed, the sequence

$$
\Lambda_{1} \stackrel{B}{\rightarrow} \Lambda_{0} \stackrel{\mathbb{1}}{\rightarrow} \mathbb{R}
$$

satisfies the property $\mathbb{1} \circ B=0$. In general, a $k$-complex $\Lambda$ is specified by a sequence of real linear spaces ${ }^{6} \Lambda_{0}, \Lambda_{1}, \cdots, \Lambda_{k}$, together with a sequence of incidence operators

$$
\Lambda_{k} \stackrel{\partial_{k}}{\rightarrow} \Lambda_{k-1} \stackrel{\partial_{k-1}}{\rightarrow} \cdots \Lambda_{1} \stackrel{\partial_{1}}{\rightarrow} \Lambda_{0}
$$

with the property that $\partial_{j-1} \circ \partial_{j}=0, j=2, \cdots, k$. The vector spaces $\Lambda_{j}, j=0,1 \cdots, k$, are called the spaces of $j$-chains. Each $\Lambda_{j}$ is generated by a finite set of $j$-cells (like edges and vertices for graphs) in the sense that $\Lambda_{j}$ is the set of functions from the $j$-cells to $\mathbb{R}$. A typical example of a $k$-complex is the triangularization of a $k$ dimensional manifold, with the $j$-cells, $j=0,1, \cdots, k$, being the sets of vertices, edges, faces, etc..

Example 2. Consider the triangularization of a 2-dimensional sphere by a tetrahedron with 4 faces, 6 edges, and 4 vertices. The matrix representation of the incidence operator $\partial_{2}$ (from the faces of the tetrahedron to its edges) is

$\begin{array}{lccccc} & \left\langle v_{1} v_{2} v_{3}\right\rangle\left\langle v_{1} v_{3} v_{4}\right\rangle\left\langle v_{1} v_{4} v_{2}\right\rangle\left\langle v_{2} v_{4} v_{3}\right\rangle \\ \left\langle v_{1} v_{2}>\right. & 1 & 0 & -1 & 0 \\ <v_{1} v_{3}> & -1 & 1 & 0 & 0 \\ <v_{1} v_{4}> & 0 & -1 & 1 & 0 \\ <v_{2} v_{3}> & 1 & 0 & 0 & -1 \\ <v_{2} v_{4}> & 0 & 0 & -1 & 1 \\ <v_{3} v_{4}> & 0 & 1 & 0 & -1\end{array}$

where the expressions $\left\langle v_{1} v_{2} v_{3}\right\rangle, \ldots$ denote the faces (with corresponding orientation), and $\left\langle v_{1} v_{2}\right\rangle, \ldots$ are the edges.

\footnotetext{
${ }^{6}$ In algebraic topology [7] one usually starts with abelian groups $\Lambda_{j}$.
} 


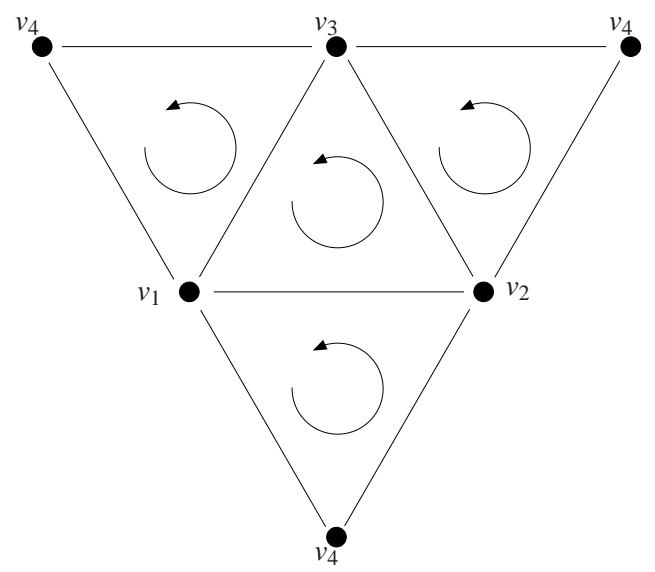

Fig. 1 Tetrahedron triangularizing a sphere

The matrix representation of the incidence operator $\partial_{1}$ (from edges to vertices) is given as

\begin{tabular}{|c|c|c|c|c|c|c|}
\hline$\left\langle v_{1}>\right.$ & -1 & -1 & -1 & 0 & 0 & 0 \\
\hline$<v_{2}>$ & 1 & 0 & 0 & -1 & -1 & 0 \\
\hline$\left\langle v_{3}\right\rangle$ & 0 & 1 & 0 & 1 & 0 & -1 \\
\hline$<v_{4}>$ & 0 & 0 & 1 & 0 & 1 & 1 \\
\hline
\end{tabular}

It can be verified that $\partial_{1} \circ \partial_{2}=0$.

Denoting the dual linear spaces by $\Lambda^{j}, j=0,1 \cdots, k$, we obtain the following dual sequence

$$
\Lambda^{0} \stackrel{d_{1}}{\rightarrow} \Lambda^{1} \stackrel{d_{2}}{\rightarrow} \Lambda^{2} \cdots \Lambda^{k-1} \stackrel{d_{k}}{\rightarrow} \Lambda^{k}
$$

where the adjoint maps $d_{j} j=0,1 \cdots, k$, satisfy the analogous property $d_{j} \circ d_{j-1}=$ $0, j=2, \cdots, k$. The elements of $\Lambda^{j}$ are called $j$-cochains.

Consider any $k$-complex $\Lambda$, with $k$-chains $\alpha \in \Lambda_{k}$ and $k$-cochains $\beta \in \Lambda^{k}$. We define, similarly as in the case of a graph (1-complex) its Kirchhoff behavior as

$$
\begin{aligned}
B_{K}(\Lambda):= & \left\{(\alpha, \beta) \in \Lambda_{k} \times \Lambda^{k} \mid\right. \\
& \left.\partial_{k} \alpha=0, \exists \phi \in \Lambda^{k-1} \text { s.t. } \beta=d_{k} \phi\right\}
\end{aligned}
$$

We will still refer to $\partial_{k} \alpha=0$ as Kirchhoff's current laws (KCL), and to $\beta=d_{k} \psi$ as Kirchhoff's voltage laws (KVL). As before, it is immediately seen that $B_{K}(\Lambda) \subset$ $\Lambda_{k} \times \Lambda^{k}$ is a Dirac structure. In particular, it follows that $\langle\beta \mid \alpha\rangle_{k}=0$ for every $(\alpha, \beta) \in B_{K}(\Lambda)$, where $<\cdot \mid \cdot>_{k}$ denotes the duality product between the dual linear spaces $\Lambda_{k}$ and $\Lambda^{k}$. 


\subsection{Open k-complexes}

Next we consider an open $k$-complex, by identifying a subset $\mathscr{V}_{(k-1)}^{b}$ of the set of all $(k-1)$-cells, called the boundary $(k-1)$-cells ${ }^{7}$, while the remaining $(k-1)$ cells are denoted as the internal $(k-1)$-cells. Define the linear space of functions from this subset of $(k-1)$-cells to $\mathbb{R}$ as $\Lambda_{b} \subset \Lambda_{k-1}$ with dual space denoted as $\Lambda^{b}$. Decompose correspondingly $\partial_{k}: \Lambda_{k} \rightarrow \Lambda_{k-1}$ as $\partial_{k}=\left(\partial_{k}^{i}, \partial_{k}^{b}\right)$, with adjoint mapping $d_{k}=\left(d_{k}^{i}, d_{k}^{b}\right)$. As before, Kirchhoff's voltage laws remain unchanged

$$
\beta=d_{k} \psi=d_{k}^{i} \psi_{i}+d_{k}^{b} \psi_{b}
$$

where $\psi_{b}$ is the vector of potentials at the boundary $(k-1)$-cells and $\psi_{i}$ is the vector of potentials at the internal $(k-1)$-cells. On the other hand, Kirchhoff's current laws are modified into

$$
\partial_{k}^{i} \alpha=0, \quad \partial_{k}^{b} \alpha=-\alpha_{b}
$$

where $\alpha_{b}$ denotes the vector of external 'currents' entering the boundary $(k-1)$ cells. By computing as before the total power we obtain for any $\alpha$ and $\beta$ satisfying $(20,21)$

$$
\begin{aligned}
& <\beta\left|\alpha>_{k}=<d_{k} \psi\right| \alpha>_{k}=<d_{k}^{i} \psi_{i}+d_{k}^{b} \psi_{b} \mid \alpha>_{k}= \\
& <\psi_{i}\left|\partial_{k}^{i} \alpha>_{k}+<\psi_{b}\right| \partial_{k}^{b} \alpha>_{k}=-<\psi_{b} \mid \alpha_{b}>_{k-1}
\end{aligned}
$$

The space of boundary variables $\left(\alpha_{b}, \psi_{b}\right) \in \Lambda_{b} \times \Lambda^{b}$ describes the distributed terminals of the open $k$-complex.

Similar to Proposition 1 it is shown that the Kirchhoff behavior of an open $k$ complex $\Lambda$ defined as

$$
\begin{aligned}
\mathscr{B}_{K}(\Lambda):= & \left\{\left(\alpha, \beta, \alpha_{b}, \psi_{b}\right) \in \Lambda_{k} \times \Lambda^{k} \times \Lambda_{b} \times \Lambda^{b} \mid\right. \\
& \left.\partial_{k}^{i} \alpha=0, \partial_{k}^{b}=-\alpha_{b}, \exists \psi_{i} \text { s.t. } \beta=d_{k}^{i} \psi_{i}+d_{k}^{b} \psi_{b}\right\}
\end{aligned}
$$

is a Dirac structure.

Analogously to graphs, Kirchhoff current laws for open $k$-complexes imply certain constraints on the incoming 'currents' $\alpha_{b}$. Indeed, by the fact that $\partial_{k-1} \circ \partial_{k}=0$ it follows that $\partial_{(k-1) b} \alpha_{b}=0$, where $\partial_{(k-1) b}$ denotes the $(k-1$-th incidence operator restricted to $\Lambda_{b} \subset \Lambda_{k-1}$. (Note that in the case of a graph the role of $\partial_{(k-1) b}$ is played by the linear map $\mathbb{1}_{b}^{T}$.) As in the case of graphs, this allows us to reduce the Kirchhoff behavior to a space that is still a Dirac structure, or, alternatively, to close the open $k$-complex. This is done by completing the open $k$-complex $\Lambda$ with space of boundary currents $\Lambda_{b}$ by an additional set of $(k-1)$-cells and $k$-cells.

Also the interconnection of open $k$-complexes is defined similar to the case of open graphs.

\footnotetext{
${ }^{7}$ One could also consider as boundary cells subsets of the $j$-th cells for $j \neq k-1$. In particular, choosing $j=k$ would correspond to 'distributed interaction'. The choice $j=k-1$ corresponds to the important case of 'boundary' interaction.
} 


\section{Port-Hamiltonian dynamics on $k$-complexes}

Consider an open $k$-complex $\Lambda$, together with its Kirchhoff behavior $\mathscr{B}_{K}(\Lambda)$. Dynamics on the $k$-complex can be defined in various ways. Similar to the case of electrical circuits we could define constitutive relations for every $k$-cell, by specifying a relation between every component of $\Lambda_{k}$ and $\Lambda^{k}$. As in the case of an electrical circuit this can be a relation of static resistive type, or a dynamic relation (of capacitive or inductive nature).

In this section we will define dynamics in a different way by specifying one type of dynamical relations between $\Lambda_{k}$ and $\Lambda^{k}$, together with resistive relations between $\Lambda_{k-1}$ and $\Lambda^{k-1}$. This will define a port-Hamiltonian dynamics, which is of relaxation type since there is only one type of physical energy (and thus no oscillations between different types of physical energy occur).

On the $k$-complex $\Lambda$, with $\partial_{k}: \Lambda_{k} \rightarrow \Lambda_{k-1}$ and $d_{k}: \Lambda^{k-1} \rightarrow \Lambda^{k}$, we define the following relations

$$
\begin{gathered}
f_{x}=-d_{k} e, \quad f_{x} \in \Lambda^{k}, e \in \Lambda^{k-1} \\
f=\partial_{k} e_{x}, \quad e_{x} \in \Lambda_{k}, f \in \Lambda_{k-1}
\end{gathered}
$$

It is checked [16] that this defines a Dirac structure $\mathscr{D} \subset \Lambda^{k} \times \Lambda_{k} \times \Lambda^{k-1} \times \Lambda_{k-1}$. This allows us to define a port-Hamiltonian dynamics by imposing the following constitutive relations. First we associate to every $k$-cell an energy storage, leading to

$$
\dot{x}=-f_{x}, \quad e_{x}=\frac{\partial H}{\partial x}(x), \quad x \in \Lambda^{k}
$$

with $H(x)$ the total stored energy, and $x \in \Lambda^{k}$ the total vector of energy variables. Furthermore, we associate to every $(k-1)$-cell a (linear) resistive relation, leading to

$$
e=-R f, \quad R=R^{T} \geq 0
$$

Substituted in (24) this yields the relaxation dynamics

$$
\dot{x}=d_{k} e=-d_{k} R f=-d_{k} R \partial_{k} \frac{\partial H}{\partial x}(x), \quad x \in \Lambda^{k}
$$

with the property that

$$
\frac{d H}{d t}=-\left(\partial_{k} \frac{\partial H}{\partial x}(x)\right)^{T} R \partial_{k} \frac{\partial H}{\partial x}(x)=-f^{T} R f \leq 0
$$

For an open complex with boundary $(k-1)$-cells the definition is modified as follows. Instead of (24) we consider 


$$
\begin{array}{cc}
f_{x}=-d_{k}\left[\begin{array}{c}
e \\
e_{b}
\end{array}\right], & f_{x} \in \Lambda^{k},\left[\begin{array}{c}
e \\
e_{b}
\end{array}\right] \in \Lambda^{k-1}, e_{b} \in \Lambda^{b} \\
{\left[\begin{array}{c}
f_{i} \\
f_{b}
\end{array}\right]=\partial_{k} e_{x},} & e_{x} \in \Lambda_{k},\left[\begin{array}{c}
f \\
f_{b}
\end{array}\right] \in \Lambda_{k-1}, f_{b} \in \Lambda_{b}
\end{array}
$$

with $f_{b}, e_{b}$ corresponding to the boundary $(k-1)$-cells, and $f, e$ corresponding to the internal cells. Imposing the same storage relations (25) and resistive relations (26) we arrive at

$$
\begin{aligned}
\dot{x} & =-d_{k}^{r} R \partial_{k}^{r} \frac{\partial H}{\partial x}(x)+d_{k}^{b} e_{b} \\
f_{b} & =\partial_{k}^{b} \frac{\partial H}{\partial x}(x)
\end{aligned}
$$

where we have split $d_{k}$ as $d_{k}=\left[d_{k}^{r} d_{k}^{b}\right]$ and $\partial_{k}=\left[\begin{array}{c}\partial_{k}^{r} \\ \partial_{k}^{b}\end{array}\right]$ (according to the division of the $(k-1)$-cells into internal cells corresponding to resistive behavior and boundary cells). This defines a port-Hamiltonian system with inputs $e_{b}$ and outputs $f_{b}$.

\subsection{Example: Heat transfer on a 2-complex}

The above formulation of systems of conservation laws and port-Hamiltonian systems on $k$-complexes will be illustrated with the model of heat transfer in a 2dimensional medium (for instance a plate). Instead of first considering the pdemodel and then discretizing, we will directly consider the dynamics on a 2-complex as arising from a triangulation of the 2-dimensional spatial domain. We assume the medium to be undeformable (hence mechanical work is neglected) and that there is no mass transfer.

We will write the heat transfer in terms of the conservation of internal energy. First we identify the physical variables as chains and cochains of the given 2complex. The components of the internal energy vector $u \in \Lambda^{2}$ denote the energy of each face. The heat conduction is given by the heat flux $f \in \Lambda^{1}$ whose components equal the heat flux through every edge. Hence the basic conservation law (conservation of energy) is given as

$$
\frac{d u}{d t}=d_{2} f
$$

The thermodynamic properties are defined by Gibbs' relation, and generated by the entropy function $s=s(u) \in C^{\infty}\left(\Lambda^{2}\right)$ as thermodynamic potential. Since we consider transformations which are isochore and without mass transfer, Gibbs' relation reduces to the definition of the vector of intensive variables $e_{u} \in \Lambda_{2}$ which is (entropy-)conjugated to the vector of extensive variables $u$ :

$$
e_{u}=\frac{\partial s}{\partial u}(u)
$$


The components $e_{u}$ are equal to the reciprocal of the temperature at each 2-face.

Since the temperature is varying over the faces, there is a thermodynamic driving force vector $e \in \Lambda_{1}$ given as the vector of differences

$$
e=\partial_{2} e_{u}
$$

By Fourier's law the heat flux is determined by the thermodynamic driving force vector as

$$
f=R\left(e_{u}\right) e,
$$

with $R\left(e_{u}\right)=R^{T}\left(e_{u}\right) \geq 0$ depending on the heat conduction coefficients. (Note the sign-difference with (26).) The resulting system is a port-Hamiltonian system (of relaxation type), with vector of state variables $x$ given by the internal energy vector $u$, and 'Hamiltonian' $s(u)$. By (28) the entropy $s(u)$ satisfies

$$
\frac{d s}{d t}=\left(\partial_{2} \frac{\partial s}{\partial u}(u)\right)^{T} R\left(e_{u}\right) \partial_{2} \frac{\partial s}{\partial u}(u)=f^{T} R\left(e_{u}\right) f \geq 0
$$

expressing the fact that the entropy is monotonously increasing. (Note again the sign-difference with the treatment above, where the Hamiltonian $H$ was decreasing.)

The exchange of heat through the boundary of the system is incorporated as above, cf. $(29,30)$, by splitting the edges (1-cells) into internal edges with the resistive relation (31) and boundary edges. This leads to

$$
\frac{d s}{d t}=\left(\partial_{2} \frac{\partial s}{\partial u}(u)\right)^{T} R\left(e_{u}\right) \partial_{2} \frac{\partial s}{\partial u}(u)+e_{b} f_{b}
$$

with $f_{b}, e_{b}$ denoting the heat flux, respectively, thermodynamical driving force, through the boundary edges.

\section{Conclusions}

A framework has been laid down for the formulation of open physical systems on $k$-complexes, generalizing the graph-theoretic formulation of electrical circuit dynamics with terminals. It has been shown that Kirchhoff's laws can be generalized to open $k$-complexes, defining a Dirac structure involving boundary currents and potentials, thus generalizing the concept of 'terminal' to the distributed case. This has been illustrated on the example of heat transfer on a 2-complex. This simple example already shows how one can directly define a finite-dimensional port-Hamiltonian dynamics, capturing the physical meaning of the involved variables and retaining the conservation laws, without the need to formulate the dynamics as a set of pde's (and possibly to discretize the pde's later on).

In future work we will apply and extend the framework to different classes of port-Hamiltonian systems on $k$-complexes (corresponding to different physical settings), and employ these models for boundary control. 
Acknowledgements It is a great pleasure to contribute to this Liber Amicorum for Professor Okko Bosgra at the occasion of his 65-th birthday. Okko has played a key role in the flourishing of the Systems \& Control community within the Netherlands, both by his scientific leadership at the Delft University of Technology, as well as by his stimulating role in bringing control engineering and mathematical systems and control theory together. I hope this chapter pays tribute to his remarkable achievements.

\section{References}

1. P. Bamberg, S. Sternberg, A course in mathematics for students of physics: 2, Cambridge University Press, 1999.

2. R.B. Bird, W.E. Stewart, E.N. Lightfoot, Transport Phenomena, John Wiley and Sons, 2002.

3. B. Bollobas, Modern Graph Theory, Graduate Texts in Mathematics 184, Springer, New York, 1998.

4. O.H. Bosgra, Lecture Notes 'Mathematical Modelling of Dynamical Engineering Systems', Delft University of Technology.

5. J. Cervera, A.J. van der Schaft, A. Banos, "Interconnection of port-Hamiltonian systems and composition of Dirac structures", Automatica, vol. 43, pp. 212-225, 2007.

6. T.J. Courant, "Dirac manifolds", Trans. Amer. Math. Soc., 319, pp. 631-661, 1990.

7. A. Dold, Lectures on Algebraic Topology, Classics in Mathematics, Springer, Berlin, 1995.

8. G. Golo, V. Talasila, A.J. van der Schaft, B.M. Maschke, "Hamiltonian discretization of boundary control systems", Automatica, vol. 40/5, pp. 757-711, 2004.

9. G. Kirchhoff, Über die Auflösung der Gleichungen, auf welche man bei der Untersuchung der Linearen Verteilung galvanischer Ströme gefürht wird, Ann. Phys. Chem. 72, pp. 497508, 1847

10. K. Kondo, M. Iri, "On the theory of trees, cotrees, multi-trees and multi-cotrees", $R A A G$ Memoirs, 2, Div. A-VII, pp. 220-261, 1958.

11. A.G.J. MacFarlane, Dynamical System Models, G.G. Harrap \& Co., London, 1970.

12. Y. Mizoo, M. Iri, K. Kondo, "On the torsion and linkage characteristics and the duality of electric, magnetic and dielectric networks", RAAG Memoirs, 2, Div. A-VIII, pp. 262-295, 1958.

13. A.J. van der Schaft, $L_{2}$-Gain and Passivity Techniques in Nonlinear Control, Lect. Notes in Control and Information Sciences, Vol. 218, Springer-Verlag, Berlin, 1996, p. 168, 2nd revised and enlarged edition, Springer-Verlag, London, 2000 (Springer Communications and Control Engineering series), p. xvi+249.

14. A.J. van der Schaft, B.M. Maschke, "The Hamiltonian formulation of energy conserving physical systems with external ports", Archiv für Elektronik und Übertragungstechnik, 49, pp. 362-371, 1995.

15. A.J. van der Schaft, B.M. Maschke, "Hamiltonian formulation of distributed-parameter systems with boundary energy flow", Journal of Geometry and Physics, vol. 42, pp.166-194, 2002.

16. A.J. van der Schaft, B.M. Maschke, 'Conservation laws and open systems on higherdimensional networks', pp. 799-804 in Proc. 47th IEEE Conf. on Decision and Control, Cancun, Mexico, December 9-11, 2008.

17. J.C. Willems, "The behavioral approach to open and interconnected systems", Control Systems Magazine, 27, Dec. 2007, pp. 46-99. 